\title{
ANALYSIS OF ENRICHED AND DEPLETED URANIUM OXIDE POWDERS BY LA-ICP-MS
}

Joshua Messerly, Nathan Saetveit, Stan Baijc, David Baldwin, and Sam Houk

Ames Laboratory USDOE

Department of Chemistry

Iowa State University

Ames, IA 50011 USA

October 2005 


\section{Introduction}

The objective of this work is to demonstrate the analysis of ensembles of small samples on filter media by laser ablation-inductively coupled plasma-mass spectrometry (LA-ICP-MS). Spatially resolved analysis of particulate samples collected on gelatinbased media is accomplished by single-shot laser ablation (using a nanosecond pulse width laser) and monitoring selected isotopes in the resulting laser ablation ICP-MS transient signal.

The capability of performing high sensitivity, spatially resolved, single shot, isotopic analyses with no sample preparation makes LA-ICP-MS valuable for the measurement of actinide isotopes at low concentrations in very small samples for nonproliferation purposes. The primary samples analyzed are an urban dust reference

material (NIST 1648) and solid uranium oxide powdered samples having different ${ }^{235} \mathrm{U}$ to ${ }^{238} \mathrm{U}$ concentration ratios. The ability to provide good detection limits and accurate isotope ratios for single laser shots is critical.

Ratios of Sn and U isotopes were determined using NIST 1648 both in a pressed pellet and on a gelatin-based substrate sprinkled with sample particles. Solid uranium oxide samples containing ${ }^{235} \mathrm{U}$ in depleted and enriched abundances were also analyzed as particle aggregates on the gelatin substrate. The depleted and enriched uranium oxide samples were nuclear reference materials (CRMS U0002 and U030-A) obtained from New Brunswick Laboratory-USDOE as well as a commercial sample of $\mathrm{UO}_{2}$. 


\section{Experimental}

Single shot laser ablation was accomplished using a CETAC Technologies LSX500 laser ablation system. This system uses a homogenized Nd-YAG laser beam, frequency-quadrupled to a wavelength of $266 \mathrm{~nm}$, with a pulse width of approximately 6 ns. The LSX-500 is capable of delivering a range of laser beam spot sizes for ablation with a user selectable variable aperture. The data in this report were acquired using a $100-$ $\mu \mathrm{m}$ laser beam spot size. A six-inch long, coiled, stainless-steel, 20-gauge tube was placed in-line between the LSX-500 and ICP-MS. The stainless-steel tube acted as a particle-size filter, minimizing the number of large particles reaching the plasma, thereby reducing spikes in the observed transient signals.

The ICP-MS instrument used was a Thermo Finnigan ELEMENT 1 magneticelectrostatic dual sector ICP-MS. This instrument was operated in low resolution mode for maximum sensitivity. The ICP RF operating power was set to $1200 \mathrm{~W}$. Other instrumental laser ablation and ICP-MS parameters were optimized on a day-to-day basis for maximum stability.

In order to simulate dust samples collected on a gel impact filter, particle aggregates were captured on a sample substrate composed of Knox Gelatin mixed with DI water. Knox Gelatin is an inexpensive gelatin with low elemental background concentrations for $\mathrm{Sn}$ and $\mathrm{U}$ and has sticking properties that make it useful for this demonstration. The ratio used was $0.75-0.80$ g gelatin to $4.5-5 \mathrm{~mL}$ of $\mathrm{DI}$ water, and the mixture was placed into a plastic weighing boat. The gelatin mixtures were heated using an IR heating lamp while mixing, then allowed to cool. Bubbles in the gelatin were skimmed off the surface with a cleaned plastic utensil before the gelatin set. In the case 
of NIST 1648, sample particles for LA-ICP-MS analysis were sprayed onto the gelatin surface using a modified membrane filtration device. Uranium oxide samples were prepared by sprinkling small amounts of uranium oxide onto the surface of the gelatin. The depleted and enriched uranium oxide samples were $\mathrm{U}_{3} \mathrm{O}_{8}$ nuclear reference materials obtained from New Brunswick Laboratory. A third, non-certified, $\mathrm{UO}_{2}$ sample was also used in this study.

\section{Results and discussion}

Previous work analyzing spatially-resolved aggregates of small samples on paperand teflon-filter media was limited by the movement of the sample as a result of recoil from the laser shot. That is, ensembles of small samples are moved by vibrations in the paper (or teflon) filter induced by the laser shockwave during ablation. Spatially resolved sample aggregates, other than the one of interest, may become entrained in the carrier gas used to transfer the ablated material to the ICP-MS. Furthermore, if another analysis of a particular sample aggregate is needed, it can be difficult to re-identify after it has moved on the filter substrate.

This work attempts to solve the particle movement problem by minimizing laser shockwave vibrations in the substrate in which the small samples are located. The sample material to be analyzed is sprinkled or sprayed onto a semi-solid gelatin substrate, rather than filter paper media. The gelatin is a sticky surface on which the particle aggregates are trapped. Since the gelatin is a semi-solid, any vibration waves from the laser shockwave are dampened. This prevents the nearby sample aggregates from becoming airborne and entrained in the LA carrier gas. Any samples not completely 
ablated by the laser are kept in place by the gelatin, allowing additional analyses if needed.

An urban dust reference material, NIST 1648, was used to develop the LA method using the gelatin substrate. NIST 1648 contains a wide variety of elements at certified concentrations. This study focused on Sn and U for method development. The concentration of $U$ in NIST 1648 is certified to be $5.5 \pm 0.1 \mathrm{ppm}$; the Sn concentration in NIST 1648 is not certified.

The results for NIST SRM 1648 are shown in Table 1 for the determination of ${ }^{118} \mathrm{Sn} /{ }^{119} \mathrm{Sn}$ and ${ }^{235} \mathrm{U} /{ }^{238} \mathrm{U}$ ratios in a pelletized sample and for aggregate samples on a gelatin media, along with literature values. Ten particle aggregates were ablated by single shots in this analysis. The measured ratios for the two elements are in good agreement between the aggregate and pellet samples. The standard deviation for the pellet analysis is slightly higher than the aggregate particle analysis because more of the sample (i.e., larger particles) is entrained in the argon sample gas, generating a slightly noisier signal. Although unconfirmed, the differences between observed and literature values for the tin isotopes may be due to an unresolved interference between ${ }^{119} \mathrm{Sn}$ and ${ }^{79} \mathrm{Br}^{40} \mathrm{Ar}$, a species that may be unintentionally formed in the argon plasma from bromine in the standard sample.

Three uranium oxide samples containing different amounts of ${ }^{235} \mathrm{U}$ were analyzed by both solution-nebulization ICP-MS and single-shot LA-ICP-MS. Two of the uranium oxide samples were certified by NBL and contained ${ }^{235} \mathrm{U}$ at $0.017 \%$ (depleted) and at $3.00 \%$ (enriched), respectively. The third uranium oxide sample analyzed was a commercial chemical material with no certified isotopic information. The ${ }^{235} \mathrm{U}$ 
concentration level of this sample was not known. The three uranium oxide samples were dissolved in a mixture of hydrochloric and nitric acids and then diluted prior to analysis. These solutions were then analyzed by ICP-MS. The solution analysis results are shown in Table II. The measured ${ }^{235} \mathrm{U} /{ }^{238} \mathrm{U}$ values of the NRMs are in excellent agreement with the NBL certified values. The ${ }^{235} \mathrm{U} /{ }^{238} \mathrm{U}$ value for the lab sample is $0.22 \%$, indicating that this sample is depleted in ${ }^{235} \mathrm{U}$. An ICP standard solution of 10 ppb natural uranium was also analyzed for comparison and yielded a ${ }^{235} \mathrm{U} /{ }^{238} \mathrm{U}$ value of $0.72 \%$. The literature value for ${ }^{235} \mathrm{U} /{ }^{238} \mathrm{U}$ in natural uranium is $0.725 \%$.

Figure 1 illustrates representative transient signals from single-shot LA-ICP-MS analysis of the uranium oxide samples. The ${ }^{235} \mathrm{U}$ and ${ }^{238} \mathrm{U}$ signal intensities are plotted on a logarithmic scale. The observed spikes in the traces are largely due to large particles being transported from the ablation cell and into the plasma. Since pure uranium oxide was analyzed, signal intensities as high as $1 \times 10^{9}$ counts were observed for ${ }^{238} \mathrm{U}$ during ablation, necessitating that the ICP-MS detection electronics be operated in analog mode. No significant background was detected for the gelatin substrate, as the analog noise was much higher than any uranium signal produced. The ${ }^{235} \mathrm{U} /{ }^{238} \mathrm{U}$ ratios were determined from these traces for the three oxide samples.

Figure 2 shows images of typical uranium oxide aggregates on the gelatin substrate before and after single-shot laser-ablation sampling. The aggregate sample in the upper half of the first image was removed and analyzed by a single laser shot. Note that the aggregate sample on the bottom half of the images remains unmoved. Particles selected for analysis were no larger than the laser-beam spot size of $100 \mu \mathrm{m}$ and no smaller than $80 \mu \mathrm{m}$. 
The results of the single-shot LA-ICP-MS analysis of the uranium oxide samples are shown in Table II. There is excellent agreement between the measured single-shot ablation values and the certified values of the NRMs and also with the solution nebulization ICP-MS results. The standard deviations in the experimental data are mainly due to irregularly sized particles (i.e., large particles), from the ablation event, entering the plasma and being observed as spikes or noise superimposed on the measured transient signal. The effect of the spikes is most notable in the measured values and standard deviations in the depleted sample, where the ${ }^{235} \mathrm{U}$ level, and hence signal, is small.

The measured single-shot LA-ICP-MS data for the uranium oxide samples were plotted against their known values. The average value of the two solution analyzes was used for the known value of the lab sample. A least squares fit of the data was performed and the results are shown in Figure 3, along with the $95 \%$ confidence interval and $95 \%$ prediction limits. The linear regression of the data yielded a correlation factor of $\mathrm{R}=0.99932$ through the data. From the figure, one can ascertain a particle that yielded a measurement of $0.725 \%{ }^{235} \mathrm{U} /{ }^{238} \mathrm{U}$ would be $95 \%$ likely to lie in the prediction range of 0.611 to $0.826 \%$. Conversely, a known natural abundance sample particle would give a measurement within the confidence range of 0.718 to $0.745 \%, 95 \%$ of the time. These prediction and confidence intervals are based on the calibration and standards used here. Additional standards over a different range could result in improved confidence and prediction probabilities. 


\section{Conclusions}

This study demonstrated the capability of LA-ICP-MS to perform single-shot analyses of particles on filter media and distinguish between them based on selected isotopic ratios. Particle aggregates of pure uranium oxide samples, having different ${ }^{235} \mathrm{U}$ to ${ }^{238} \mathrm{U}$ concentration ratios, and an urban dust standard (NIST 1648), containing natural uranium at $5 \mathrm{ppm}$, were analyzed on a gelatin substrate. There is excellent agreement between the measured single-shot ablation ${ }^{235} \mathrm{U} /{ }^{238} \mathrm{U}$ values and the certified values of the NRMs and also with the solution nebulization ICP-MS results.

This study shows that single-shot LA-ICP-MS can readily differentiate between very small samples of depleted, natural, and enriched uranium containing samples. From the reported data, a known natural abundance sample particle would yield a measurement within the confidence range of 0.718 to $0.745 \%, 95 \%$ of the time. Similarly, a sample that resulted in a measured vaule of $0.725 \%{ }^{235} \mathrm{U}$ would be $95 \%$ likely to lie in the prediction range of 0.611 to $0.826 \%$, based on the calibration and standards used here.

\section{Acknowledgement}

Ames Laboratory is operated for the USDOE by Iowa State University under Contract No. W-7405-Eng-82. This work was supported by the USDOE, Office of Defense Nuclear Nonproliferation, and the Office of Nonproliferation Research and Engineering, NA-22. 
Table I. Results for NIST SRM 1648. Ten shots averaged for each analysis. (1 s. d. uncertainties in last digit in parentheses)

\begin{tabular}{c|c|c|c}
\hline & Pelleted Sample & Particle-Gelatin Sample & $\begin{array}{c}\text { Literature } \\
\text { Ratio Value }\end{array}$ \\
\hline $\begin{array}{c}\text { Measured } \\
{ }^{118} \mathrm{Sn} /{ }^{19} \mathrm{Sn} \text { Ratio } \\
\begin{array}{c}\text { Measured } \\
{ }^{235} \mathrm{U} /{ }^{238} \mathrm{U} \text { Ratio }\end{array}\end{array}$ & $2.7(5)$ & $2.6(1)$ & 2.82 \\
\hline
\end{tabular}


Table II. Results for Uranium Samples (1 s. d. uncertainties in last digit in

\begin{tabular}{|c|c|c|c|}
\hline & $\begin{array}{l}\text { Laser Ablation } \\
\text { Measurement } \\
{ }^{235} \mathrm{U} /{ }^{238} \mathrm{U} \%\end{array}$ & $\begin{array}{c}\text { Solution } \\
\text { Measurement } \\
{ }^{235} \mathrm{U} /{ }^{238} \mathrm{U} \%\end{array}$ & Certified Value \\
\hline Depleted Standard & $\begin{array}{c}0.020(2) \\
\text { (avg. of } 29 \text { shots) }\end{array}$ & $0.0176(6)$ & $0.01755(5)$ \\
\hline & $\begin{array}{c}0.23(1) \\
\text { (avg. of } 29 \text { shots) } \\
--\end{array}$ & $\begin{array}{c}0.219(5) \\
\text { (dissolution 1) } \\
0.23(1) \\
\text { (dissolution 2) }\end{array}$ & $--^{a}$ \\
\hline Enriched Standard & $\begin{array}{c}3.15(9) \\
\text { (avg. of } 30 \text { shots) }\end{array}$ & $3.11(6)$ & $3.137(2)$ \\
\hline $\begin{array}{l}\text { Natural 10ppb } \\
\text { Standard }\end{array}$ & -- & $0.72(4)$ & $0.725^{\mathrm{b}}$ \\
\hline
\end{tabular}

a. No certified value was available for this sample. The sample was obtained from our laboratory inventory.

b. Handbook of Chemistry and Physics, $68^{\text {th }}$ Edition, CRC Press (1988). 

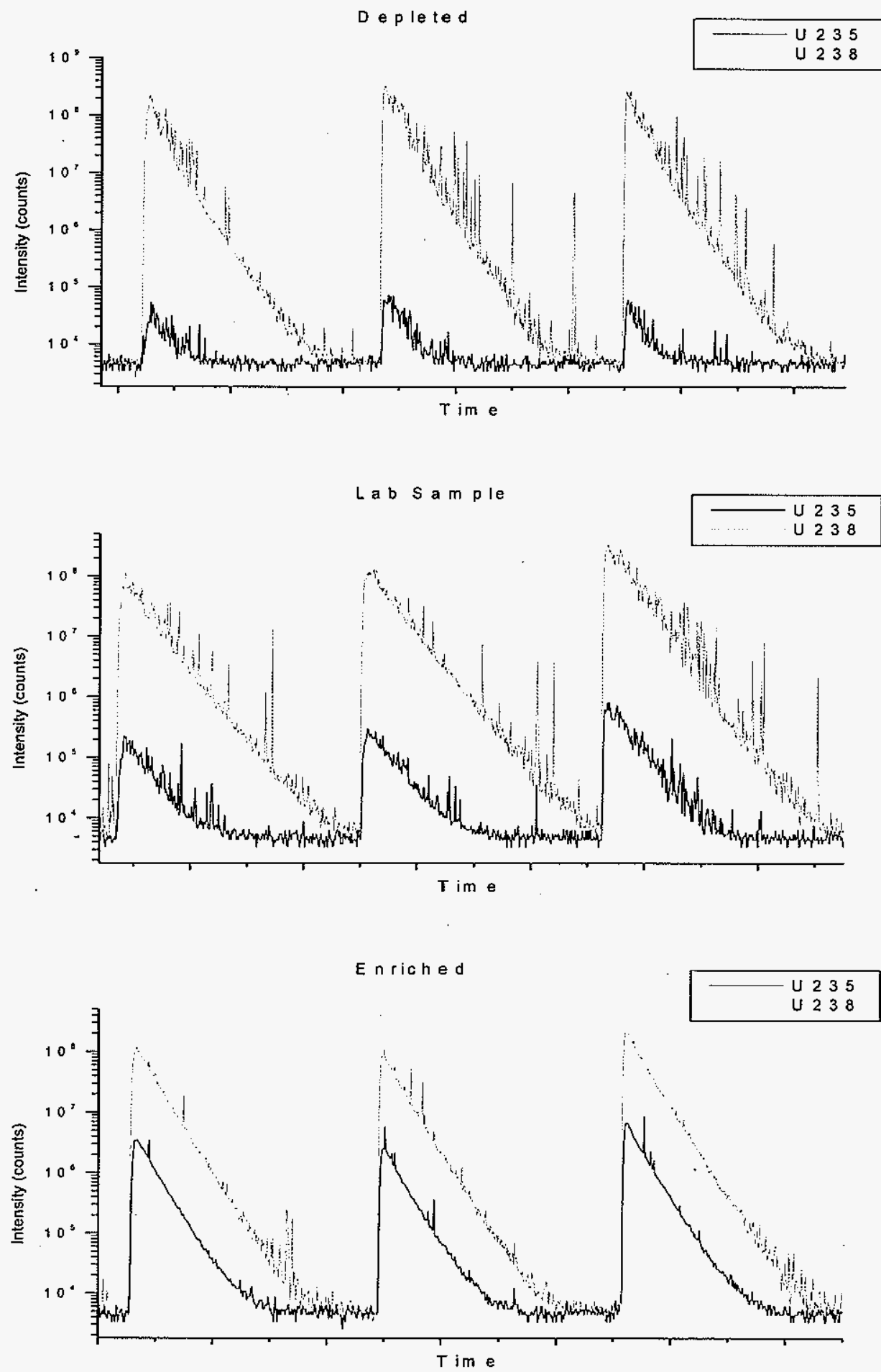

Figure 1. Typical transient signals from single-shot LA-ICP-MS analysis of delpete, lab, and enriched uranium oxide samples. 

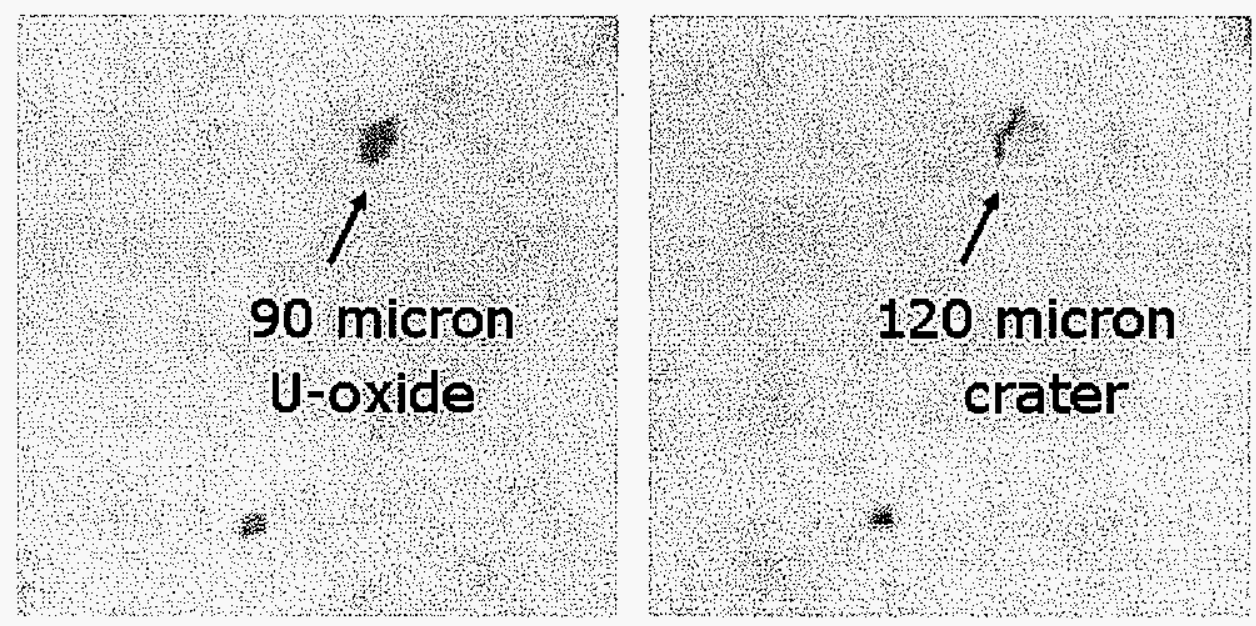

Figure 2. Images of uranium oxide particles on a gelatin substrate before (left) and after (right) single-shot laser ablation sampling. 


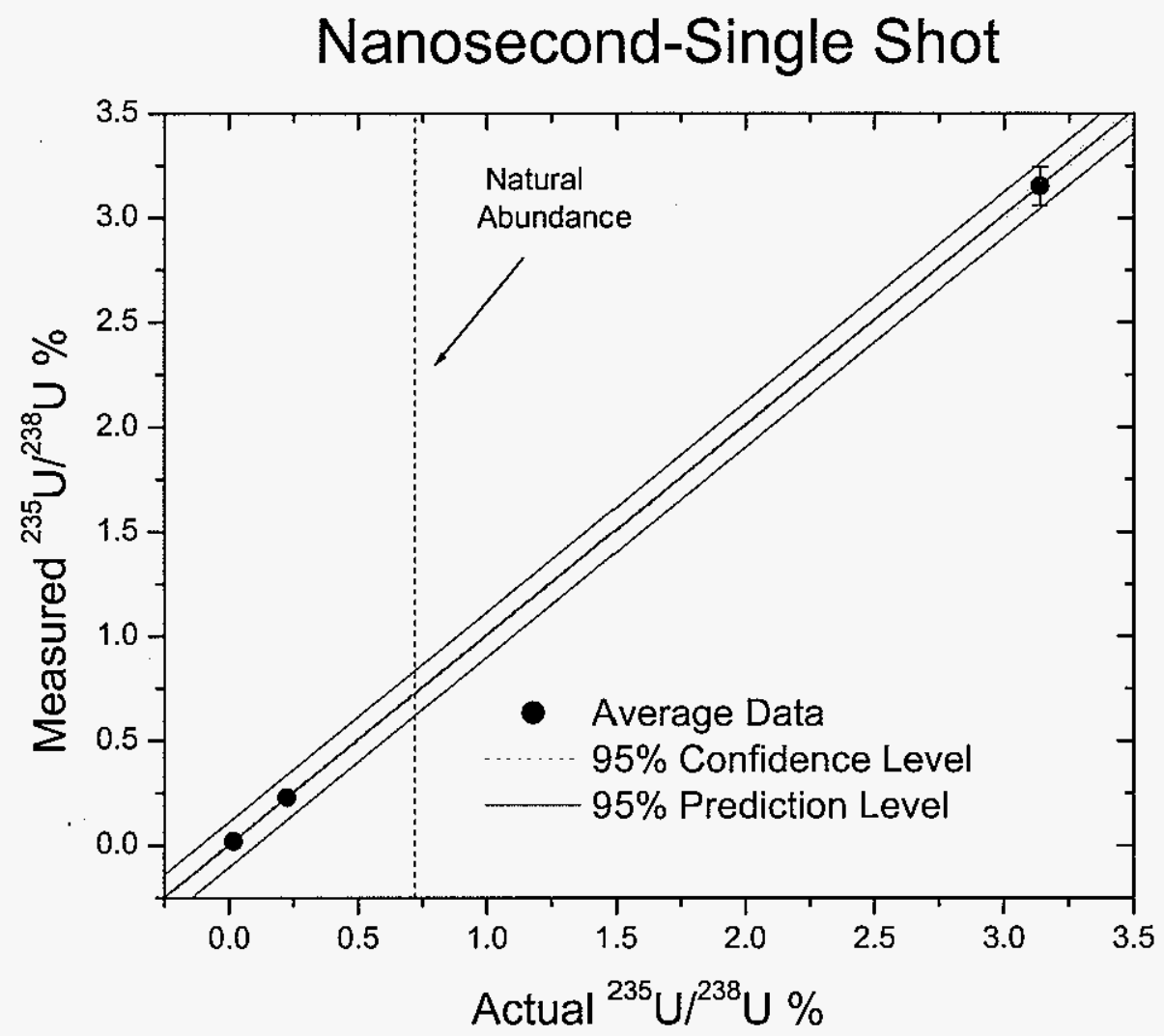

Figure 3. Linear regression fit of uranium oxide on gelatin samples, analyzed by single-shot LA-ICPMS. 